\title{
Estado del arte de la investigación sobre argumentación y escritura multimodal desde una perspectiva didáctica*
}

\author{
María Elicenia Monsalve Upegui**
}

\begin{abstract}
Resumen
Esta ponencia revisa y analiza algunas investigaciones sobre la enseñanza de la argumentación en la escritura de textos multimodales digitales, temática que se aborda en tres momentos a saber: 1) argumentación y didáctica de los años 60 al 90, 2) enseñanza de la argumentación desde una perspectiva cognitiva y epistémica desde los 90 al 2000; y 3) formas de argumentar apoyadas en recursos multimodales desde los años 90 al 2013. Finalmente, se presentan las conclusiones enfocadas en develar algunas problemáticas que abren el campo de discusión e investigación.
\end{abstract}

Palabras clave: argumentación, escritura, multimodalidad, didáctica.

\section{State of the art in research about argumentation and multimodal writing from a didactic perspective}

\begin{abstract}
This is a revisión and an analysis of some research work about argumentation teaching in the writing of digital multimodal texts, a subject approached in three moments, as follows: 1). Argumentation and didactics between the decades of the 60's and the 90 's; 2). Argumentation teaching from cognitive and epistemic focuses from the 90 's to the 2000 and
\end{abstract}

3). Argumentation forms supported on multimodal resources in the years between 1990 and 2013. The conclusions are finally presented, focusing on revealing some problems that open the discussion and research field.

Key words: argumentation, writing, multimodality, didactics.

\section{Estado da arte da investigação sobre argumentação e escritura multimodal desde uma perspectiva didática}

\section{Resumo}

Esta conferência revisa e analisa algumas investigações sobre o ensino da argumentação na escritura de textos multimodais digitais, temática que se aborda em três momentos a saber: 1) argumentação e didática dos anos 60 aos anos 90 , 2) ensino da argumentação desde uma perspectiva cognitiva e epistémica desde os anos 90 ao ano 2000; e 3) formas de argumentar apoiadas em recursos multimodais desde os anos 90 ao ano 2013. Finalmente, apresentam-se as conclusões enfocadas em desvendar algumas problemáticas que abrem o campo de discussão e investigação.

Palavras chaves: argumentação, escritura, multimodalidade, didática.

\footnotetext{
* Este artículo hace parte de los avances relacionados con la revisión de literatura especializada, en el marco de los estudios doctorales desarrollados en la Universidad de Antioquia Colombia. Se presenta una aproximación al estado del arte de la investigación en cuestión.

** Magíster en Educación. Estudiante del Doctorado en Educación y Profesora del Programa de Integración de Tecnologías a la Docencia, Integrante del Grupo de Investigación Didáctica y Nuevas Tecnologías. Universidad de Antioquia, Medellín (Colombia).
} 


\section{Introducción}

Aproximadamente desde el año 70, con el trabajo de Perelman (1977), se ha considerado la argumentación como una forma de convencer con razones o persuadir a un auditorio. Toulmin (1958), en sus planteamientos, considera que abordar la argumentación desde la lógica formal es insuficiente para describir su funcionamiento, así como su discusión crítica en el marco de las interacciones cotidianas. Además, se inician los estudios sobre la argumentación desde una perspectiva pragmadialéctica propuesta por Van Eemeren y Grootendorst (2002), quienes consideran la argumentación como una posibilidad para resolver una diferencia de opinión, y su principal interés radica en llegar a acuerdos con la contraparte. En esta última teoría se busca fomentar una actitud propicia para la discusión, ya que hay un interés por las situaciones argumentales en tanto estas permiten establecer unas condiciones ideales para que se dé la interacción.

Las teorías mencionadas dieron paso a una amplia variedad de líneas de investigación en campos como lingüística, filosofía, ciencias naturales, matemáticas y educación. Recientemente, ha surgido un interés particular desde campos como la lingüística (Kress, 19771987), la semiótica social de Halliday (1978) y la gramática visual de Kress y van Leeuwen (1996) en abordar la argumentación mediada por recursos multimodales como la imagen estática o en movimiento, el audio, el vídeo, el color, y otros recursos que las tecnologías de la información y las comunicaciones han aportado.

En el nivel didáctico, la argumentación y el uso de recursos multimodales son objetos de estudio relevantes, dado que están presentes en las diversas áreas curriculares que se proponen para la enseñanza. Las investigaciones de Wikan, Mølster, Bjørn y Hope (2010); Jewitt, (2002); Weinberger y Fischer (2006) han indagado cómo el uso de recursos multimodales en la escritura y la comunicación permite argumentar de modo más eficaz y de una manera distinta al discurso oral.

Las investigaciones de Arango-Lasprilla, Sosa-Gallego y Hurtado-Vergara, (2008);
Dolz (1993-2011); Dolz y Camps (1989-2004), Jiménez-Alexandrie (2007); Kuhn (1991) y Tamayo- Alzate, (2013) , Daly y Unsworth, (2011); Stein, (2008); Jiménez-Aleixandre y Díaz de Bustamante, (2003) han abordado la argumentación en diversas áreas curriculares, con algunas similitudes en términos de estrategias pedagógicas y didácticas a saber: discusiones sobre algún tema en particular, proyectos de aula investigativos, y debates en clase. Además, las investigaciones se han interesado en evaluar procesos de escritura, el discurso oral, habilidades cognitivas de alta complejidad, o también se han enfocado en abordar temáticas escolares. Este tema, ha sido investigado con estudiantes de la Educación Básica Primaria, Secundaria y Educación Superior. Con resultados que indican que, los estudiantes de la educación básica primaria y secundaria presentan serios problemas para argumentar y comprender los discursos argumentativos en diversos contextos.

Esta ponencia analiza algunas teorías que explican el concepto de argumentación, y presenta un recorrido investigativo que da cuenta de cómo se ha abordado la argumentación en los últimos veinte años. Este recorrido se organiza en tres momentos que identifican los énfasis que han caracterizado los estudios revisados.

\section{Momento 1: Argumentación y didáctica}

En las décadas del 60 al 90, se difundieron enfoques teóricos que responden a tradiciones investigativas de orden epistemológico; cada una de ellas ha proporcionado aportes en cuanto a formas, metodologías y conceptos para abordar la investigación didáctica en argumentación. En el marco de la retórica de Perelman y Olbrechts-Tyteca (2001), se plantea “(...) para que haya argumentación, es necesario que, en un momento dado, se produzca una comunidad efectiva de personas. Es preciso que se esté de acuerdo, ante todo, en la formación de esta comunidad intelectual $y$, después, en el hecho de debatir juntos una cuestión determinada" (p. 48), lo que significa que debe existir aceptación y atribución de valor a la adhesión del interlocutor, el uso de un lenguaje común y la apropiación conceptual, evitando los dogmas y buscando más bien como 
propósito llevar a los participantes a razonar y a tener en cuenta los discernimientos del otro dentro de la comunidad, con el objetivo de que los interlocutores se construyan en relación con el otro en un marco ético y, sobre todo, en el marco de las claridades conceptuales (Popper 1982).

Para Toulmin (1958), la argumentación se refiere a la capacidad de exponer una tesis, controvertirla, examinar sus consecuencias, intercambiar pruebas, y plantear buenas razones que lleven a una conclusión. En este orden de ideas la argumentación requiere de un conjunto de habilidades, capacidades, disposiciones y conocimientos logrados mediante el aprendizaje, lo cual le permite a un sujeto la producción de argumentos en contextos diversos y con fines particulares. Toulmin centra su teoría en el desarrollo de un modelo argumental utilizado para evaluar la producción de argumentos.

Si bien estas teorías han dado línea para explorar el campo de la argumentación, también ofrecen estructuras y modelos que al momento de aplicarlos en la educación Básica Primaria, se quedan cortos en tanto las condiciones y contextos de enseñanza demandan de elementos didácticos y pedagógicos que permitan a los estudiantes el aprendizaje de la argumentación en situaciones auténticas y cotidianas relacionadas con aprender para la vida y para la constitución de un sujeto crítico, democrático y reflexivo.

En los últimos años, se han encontrado investigaciones en el ámbito latinoamericano, que se inscriben en el campo de la enseñanza de la argumentación, como las desarrolladas por Parodi-Sweis (2000); Poblete-Olmedo (2005); Arango-Lasprilla, Sosa-Gallego y Hurtado-Vergara (2008), Caballero-Escorcia y Hurtado-Vergara (2008) y Ramírez-Guzmán (2012), quienes han estudiado la comprensión y producción de textos argumentativos orales y escritos en las aulas de clase. Otras se enfocan en indagar por aspectos discursivos y lingüísticos (Martínez-Solís, 2002). Las investigaciones de Cros-Alavedra (19952003); Del Caño, (1999); Perelman (2001); Plantin (1998); Weston, (1999); Ducrot y Anscombre, 1994) se centraron en el estudio del discurso argumentativo como proceso dialógico y de conocimiento, o en la indagación de los mecanismos o estrategias discursivas y lingüísticas que utilizan los hablantes para argumentar.

En las investigaciones descritas se presenta cierta similitud en las propuestas educativas para la enseñanza de la argumentación desde una perspectiva clásica enfocada en la producción escrita de textos argumentativos que apelan a unas reglas de carácter lingüístico y cognitivo. Si bien se identifican elementos relacionados con prácticas que se interesan en aportar a la construcción de sociedades democráticas, justas y pacíficas, a la adquisición de conocimientos culturales, sociales y científicos, los mecanismos para lograr dichos aportes no se integran a prácticas globales que respondan a las exigencias de un mundo permeado por el uso de tecnologías de la información y comunicaciones -TIC- con diversas posibilidades para la escritura, el debate, la participación y la construcción social del conocimiento.

En este sentido, algunas investigaciones han demostrado que el uso de recursos y aplicaciones provenientes de las TIC como soporte para el aprendizaje aumentan las interacciones entre pares y el desarrollo de habilidades argumentativas; se mejora la comprensión y la resolución de problemas, y se fortalece la reflexión crítica (Collins y Collins, 1996; Ward y Tiessen, 1997; Golanics, y Nussbaum, 2007).

\section{Momento 2: Enseñanza de la argumentación desde una perspectiva cognitiva $y$ epistémica}

De los años noventa al dos mil, se produce un giro interesante en las investigaciones relacionadas con la argumentación, indagando por las habilidades para argumentar a través de variables cognitivas, epistémicas y del contexto. Estudios recientes se han interesado por explorar las habilidades que utilizan los estudiantes para argumentar, entre ellas, la razonabilidad, la contrargumentación y la reflexión crítica (Felton y Khun, 2001; Voss, Wiley y Sandak, 1999; Kuhn, 1991; Voss, Blais, Means, Greene y Ahwesh, 1986; Marinkovich, 
2007; Monsalve-Upegui, 2013). Estos estudios coinciden en afirmar que las habilidades exploradas son complejas para enseñar en la escuela. Las investigaciones convergen en que los adolescentes y jóvenes presentan serias dificultades para contrargumentar, por cuanto son menos expuestos a situaciones que así lo exijan.

Desde la perspectiva cognitiva un grupo de investigaciones se ha interesado en indagar por las funciones de la argumentación entre las que se encuentran: la función social sustentada en los planteamientos de Vigostky (1978) y Kuhn (1991); la función dialéctica, propuesta Van Eemeren y Grootendorst (2002); la función cognitiva planteada por Kuhn (1991), y la función epistémica sustentada en los planteamientos de Leitão (2000), Kuhn (1991), Baker (1999), Nussbaum y Bendixen (2003), y Andriessen, Baker y Suthers (2003) Andriessen (2006). Desarrollar estas funciones en el proceso de aprendizaje permite que los estudiantes pongan en acción una serie de habilidades o destrezas que les permiten asumir posiciones, dirimir conflictos, resolver problemas de diversas índole, reflexionar críticamente sobre áreas o conocimientos disciplinares y sobre sus propios actos, conocimientos y transformar los saberes previos en conocimientos nuevos.

Los estudios explorados en este apartado convergen respecto a la argumentación como una habilidad central en la construcción del conocimiento dado que permite a los estudiantes acercarse a los fenómenos, estudiarlos, cuestionarlos y reflexionar sobre sus propias acciones, confrontar sus conocimientos previos con las teorías que circulan, desarrollar la capacidad de juicio, contradecir sus propias opiniones y las de otros, razonar, pensar críticamente, y comprender epistemológicamente adicionar objetivos del conocimiento.

\section{Momento 3: formas de argumentar apoyadas en recursos multimodales}

Entre los años noventa a la fecha, se introducen intereses por la función de la imagen en la argumentación y posteriormente se presentan investigaciones que indagan por la argumentación apoyada en recursos audiovisuales multimodales (imagen, audio, vídeo, texto, etc.). Se integran en las investigaciones aspectos de orden conceptual y didáctico que buscan tratar problemáticas en la enseñanza de la argumentación de forma integral y acordes con las demandas de la sociedad actual o "sociedad del conocimiento".

a) En el orden de lo conceptual, están los estudios centrados en caracterizar y conceptualizar sobre la argumentación visual, entre ellos, Groarke (1996); Blair; (1996-2004); Fleming (1996); Nettel (2005); Fisher (2005); Birdsell y Groake (2006); Dove (2012); Van den Hoven (2012); y Roque (2012). El trabajo de los teóricos citados parte de una diversidad de disciplinas que están interesadas en explorar de forma sustancial la retórica visual a través del análisis de fotografías, dibujos, gráficos, tablas, e imágenes en movimiento; además, exploran las maneras como son utilizados los elementos visuales para influir en las actitudes de las personas, sus creencias y opiniones. Los argumentos visuales se refieren a premisas y conclusiones tradicionales representadas en imágenes; ahora bien, para Blair (1996), son argumentos expresados visualmente.

Se presentan en las definiciones mencionadas dos elementos importantes: "argumentos" "visuales". En primera medida, los argumentos por tradición han sido considerados de naturaleza retórica y se dan mediante la verbalización; sin embargo, algunas conceptualizaciones recientes incluyen el tema de lo visual como un modo de argumentar. Por ejemplo, Blair (1996) menciona que los "argumentos visuales han de entenderse como argumentos proposicionales y su función argumentativa se expresa visualmente" ( $p$. 26). En este sentido, lo visual no sería solo una ilustración del argumento verbal, sino que contiene una estructura argumental organizada y clara. Los argumentos visuales también han sido considerados como una concatenación de declaraciones visuales dadas en una imagen en particular, la cual puede funcionar como una buena razón para sustentar un argumento (Groarke, 1996).

b) Los estudios de Collins y Collins (1996); Ward y Tiessen, (1997); Veerman (2003) han explorado el uso de recursos TIC para la 
enseñanza de la argumentación, indagando por las discusiones y el aprendizaje de orden colaborativo, y la construcción y el intercambio de argumentos con el objetivo de promover el aprendizaje. Estos estudios han demostrado que el uso de herramientas informáticas como soporte para el aprendizaje aumenta las interacciones entre pares y el desarrollo de habilidades argumentativas, dado que a través de las discusiones online se mejoran la comprensión y la resolución de problemas, y se fortalece la reflexión crítica (Nussbaum y Bendixen 2003; Weinberger y Fischer, 2006; Noroozi, Weinberger, Biemans, Mulder y Chizari 2013).

Los estudios mencionados evidencian que a la hora de diseñar estrategias para fomentar la argumentación apoyada en TIC se deben tener en cuenta elementos que garanticen el éxito de las mismas, a saber: dar instrucciones claras a los estudiantes, elegir plataformas o software con interfaces dinámicas y con elementos gráficos que permitan a los estudiantes apoyar sus argumentos, el profesor debe ser un mediador en las discusiones o diálogos de los estudiantes y finalmente, plantear temas de interés que motiven los participantes a la argumentación. Como puntos de convergencia, las investigaciones plantean la argumentación como una habilidad cognitiva a través de la cual se llega a la resolución de problemas, y se construye y se adquiere el conocimiento.

Sin embargo, es importante tener en cuenta los planteamientos de Andriessen, (2006); Veerman, (2003); y Koschmann, (2010), dado que advierten sobre algunas limitaciones que presenta el uso de tecnologías para el desarrollo de discursos argumentativos, entre ellas: desorganización e incoherencia en el discurso que presenta incoherencia, poco uso de los recursos para la reflexión y la contrargumentación, así como abandono de las discusiones si son interpelados por sus pares discutidores.

Es recurrente evidenciar en las investigaciones abordadas el interés y la importancia de abordar la argumentación en la escuela indistintamente de las herramientas a utilizar o de los temas objeto de discusiones y las formas de trabajarlos (debates o escritura). Los aportes de las investigaciones presentadas son importantes para la didáctica de la argumentación en cuanto evidencian la necesidad de abordar el tema con estrategias de enseñanza en las que se utilicen diversos medios y recursos, como también explorar en los recursos multimodales otras formas para argumentar. Por otro lado, se pone en evidencia que la argumentación está altamente asociada a las concepciones de aprendizaje desde la construcción social de significados, de la interacción, la reflexión y la argumentación, lo cual se contrapone a los modelos tradicionales para la enseñanza abordada desde los métodos inductivos.

Los estudios sobre multimodalidad abordados desde 1991, en el ámbito anglosajón, se interesan en el análisis discursivo y lingüístico, y se vinculan al campo educativo mediante la evaluación de recursos como libros de texto, o multimedios constituidos por recursos multimodales abordados en la escuela desde diferentes visiones. Los intereses que orientan las investigaciones y las teorías al respecto se han circunscrito en diferentes áreas del conocimiento, entre ellas ciencias naturales, matemáticas, e inglés y lenguaje en menor proporción. Esta investigación ha centrado sus intereses en estudiar cómo el uso de recursos multimodales en la educación transforma las prácticas de escritura, el acceso al conocimiento y la representación del mismo mediante diferentes modos de representar las ideas.

Las teorías y las investigaciones realizadas por Kress y van Leeuwen, (1996); New London Group, (1996); Kress y Benzemer (2008); Daly y Unsworth (2011); Kress (2005), Burn y Parker's (2001); Jewitt, (2008); Stein, (20002008) han indagado cómo los estudiantes exploran significados a través de los diferentes recursos semióticos disponibles en pantalla y lo que significa esto para el aprendizaje. La investigación sobre la producción de textos multimodales ha explorado su potencial en el significado y estructura de los textos, a través de la vinculación de recursos semióticos, como ocurre con el hipertexto y el hipermedio (Jewitt, 2002). La importancia del concepto de multimodalidad al momento de pensar en la producción de textos, en los contextos de 
aprendizaje escolar, radica en el surgimiento de nuevos modos de representación y comunicación de las ideas. Por tanto, las prácticas de escritura multimodal han dado señales de un cambio sustancial en el tema de la alfabetización. Una muestra de ello es la facilidad con que los estudiantes pueden dibujar, pintar, cantar y bailar, además de producir sus propias películas digitales, dominar juegos complejos de computador y participar en la creación de sitios web interactivos.

Los estudios de Archer (2010), Shin-shin y Cimasko (2008), de Daly y Unsworth (2011), Wikan, Mølster, Bjørn y Hope (2010), MonsalveUpegui (2013) han indagado por la producción y comprensión de textos argumentativos multimodales. Dichas investigaciones se han preguntado por la relación entre los elementos multimodales y la construcción del conocimiento, por el significado que tiene para los estudiantes de la Educación Superior la escritura de un texto académico con características multimodales, y por las habilidades que utilizan los estudiantes en la producción de por dichos textos en la Educación Básica. Los resultados de las investigaciones mencionadas evidencian la necesidad de definir criterios claros para la producción de textos académicos multimodales, enfocados en contextos particulares, con propósitos específicos, y pensar los vínculos del texto con los modos de representación, como también pensar en la innovación y en el uso de los recursos a utilizar.

Las investigaciones descritas coinciden en la importancia de hacer un llamado a los docentes para motivar la atención de los estudiantes respecto a analizar cómo se relacionan las imágenes entre sí, los textos y la interacción de los significados que de estas puedan surgir. Porque si bien los estudiantes logran establecer relaciones entre el lenguaje-imagen, y otros recursos no debe suponerse que el hecho de comprender estas relaciones es un gran logro para conectar dos partes diferentes de un texto, ya que los estudiantes pueden necesitar apoyo para de-construir estructuras gramaticales complejas que ayuden a comprender las relaciones entre dichas partes.

De los estudios mencionados, se destacan como importantes los siguientes elementos: las representaciones utilizadas por los estudiantes tienen una estrecha relación con el conocimiento, su construcción y su producción en el ámbito educativo; las representaciones son procesos de construcción que implican elementos epistemológicos y metodológicos desarrollados mediante, la interacción social, y las relaciones establecidas con los objetos de conocimiento. Estos elementos están relacionados con los planteamientos derivados de las teorías constructivistas que se sustentan en los aportes de Vigotsky, (1988) y Martí, (2012), quienes argumentan que las personas construyen los objetos del conocimiento de acuerdo con sus realidades y con sus estructuras cognitivas, lo cual implica que sus construcciones estén mediadas por condiciones históricas, sociales y culturales.

\section{A modo de conclusiones}

De forma relevante, persisten en los reportes investigativos las dificultades relacionadas con el aprendizaje y la enseñanza de la argumentación en la escuela, complejidad evidenciada en los diferentes marcos conceptuales que intentan comprender conceptos, metodologías y procedimientos relacionados con el objeto de estudio presentado, así como en la falta de propuestas y orientaciones claras a los docentes acerca de cómo enseñar a los estudiantes a argumentar desde edades tempranas. Estas dificultades han sido reconocidas por autores como Martínez-Solís (2001); Khun (1991); Perelman (2001); Rubio y Arias (2002); Sánchez y Álvarez (2001); Andriessen, Baker, y Suthers (2003). Además, estos autores insisten en la importancia de investigar acerca de cómo argumentan los estudiantes en diversos contextos y en situaciones cotidianas, de tal forma que ofrezcan mayores claridades para orientar los procesos de enseñanza.

Aportes importantes en la búsqueda de marcos comprensivos se reconocen en los trabajos realizados sobre cómo los estudiantes construyen el conocimiento mediante procesos de argumentación apoyados en el uso de herramientas TIC y sin estas (Felton y Khun, 2001; Jiménez-Alexandrie 2007; Weinberger, Stegmann, y Fischer, 2007; Ramírez- Guzmán, 
2012). Estos autores han mostrado las debilidades que presentan los estudiantes en la construcción del conocimiento, en las habilidades argumentativas, y también en las propuestas curriculares utilizadas para enseñar a argumentar en la Educación Básica. Por lo tanto, se evidencia la necesidad de trascender la retórica a marcos de análisis orientados a la construcción del conocimiento en situaciones auténticas de aprendizaje, indagando por aspectos epistemológicos, sociales, cognitivos y didácticos.

Los estudios sobre la producción de textos argumentativos digitales con características multimodales y las conceptualizaciones sobre argumentación visual, propuestos por (Dove, 2012; Van den Hoven, 2012; y Roque, 2012; Archer 2010; Shin-shin y Cimasko, 2008; de Daly y Unsworth, 2011), si bien son incipientes, dejan abierto un campo importante para la investigación en las aulas de clase y para la transformación en las prácticas de enseñanza de la argumentación, la construcción del conocimiento mediado por elementos multimodales y sobre cómo potenciar las habilidades argumentativas en los estudiantes de la Educación Básica.

Comprender de una mejormanera las relaciones entre conceptos, las rupturas teóricas, las proximidades didácticas dadas entre unas investigaciones y otras en periodos distintos, los intereses por trascender las teorías netamente retóricas en la argumentación y las filiaciones entre unas y otras prácticas de enseñanza de la argumentación en la escuela, permiten preguntarse: ¿de qué manera construyen los estudiantes el conocimiento cuando escriben utilizando recursos multimodales? ¿Qué factores epistemológicos, sociales, y didácticos influyen en la construcción del conocimiento de los estudiantes cuando escriben utilizando recursos multimodales? ¿Cuál es lugar de la construcción del conocimiento apoyado en recursos multimodales, en la vida cotidiana de los estudiantes?

Interrogantes como los aquí planteados fomentan el desarrollo de investigaciones empíricas y teóricas sobre aspectos cognitivos, epistemológicos y didácticos relacionados con la enseñanza o el aprendizaje en la construcción del conocimiento argumentativo en escenarios de escritura multimodal, y contribuyen a la diversificación de propuestas, y análisis sobre cómo se podrían transformar las prácticas en la enseñanza de los objetos de estudio presentados en este texto.

\section{Referencias bibliográficas}

- Andriessen, J., Baker, M y Suthers, D. D (2003). Arguing to learn: confronting cognitions in computer supported collaborative learning environments. Dordrecht Boston: Kluwer Academic Publishers.

- $\quad$ Andriessen J. (2006). Collaboration in computer conferencing. En Collaborative Learning, Reasoning, and Technology (Eds. A. M. O’Donnell, C. E. Hmelo-Silver y G. Erkens pp. 197-230. Chap. 9), Erlbaum, Mahwah, NJ.

- Arango-Lasprilla, Sosa-Gallego y HurtadoVergara (2008). Comprensión lectora de los textos argumentativos en los niños de quinto grado de educación básica primaria Trabajo de Investigación para optar al título de Magíster en Educación. Medellín: Universidad de Antioquia. Dirigió cómo Tutor, Rubén Darío Hurtado Vergara.

- $\quad$ Archer, A. (2010). Multimodal texts in Higher Education and the implications for writing pedagogy. English in Education, 44(3), 201213.

- Baker, M. J. (1999). Argumentation and Constructive Interaction. En: G. Rijlaarsdam y E. Espéret (Series Eds.) y Pierre Coirier and Jerry Andriessen (Vol. Eds.) Studies in Writing Foundations of Argumentative Text Processing, 179-202. Amsterdam: University of Amsterdam Press.

- $\quad$ Blair, J. A. (1996). The possibility and actuality of visual arguments. Argumentation and advocacy, 33, 23-39.

- Blair, J. A. (2004). The Rhetoric of Visual Arguments, En: C. A. Hill, y Helmers, M. (Eds). Defining visual retorics. (Chapter 2: pp, 41-61). Mahwah: Lawrence Erlbaum Associates.

- $\quad$ Birdsell, D. S. y Groake, L. (2006). Outlines of a theory of visual argument. Argumentation and Advocay, 43, 103-113.

- Burn, A. y Parker's, D. (2001). 'Making your Mark: digital inscription, animation, and a new visual semiotic', Education, Communication \& Information, 1(2), 155-179. 
- Caballero-Escorcia y Hurtado-Vergara (2008). Comprensión lectora de los textos argumentativos en los niños de poblaciones vulnerables escolarizados en quinto grado educación básica primaria. Trabajo de Investigación para optar al título de Magíster en Educación. Medellín: Universidad de Antioquia. Dirigió cómo Tutor, Rubén Darío Hurtado Vergara.

- Camps, A. (1989). Modelos del proceso de redacción: algunas implicaciones para la enseñanza. Infancia y aprendizaje, 49, 3-19.

- Camps, A. (1995). Aprender a escribir textos argumentativos: características dialógicas de la argumentación escrita. Comunicación, lenguaje y educación, 26, 51-64.

- Camps, A. (1996). Proyectos de lenguas entre la teoría y la práctica. Cultura y aprendizaje, 2 , 43-57.

- Camps, A. (2003). Proyectos de lengua entre la teoría y la práctica. En. A. Camps (Ed.) Secuencias didácticas para aprender a escribir. Barcelona: Graó.

- Camps, A. (2004). Objeto, modalidades y ámbitos de la investigación en didáctica de la lengua. Lenguaje, 32, 7-27

- Collins, C., y Collins, S. (1996). The Internet as a tool. National Educational Computing Conference 1996, Minneapolis, MN.

- Cros-Alavedra, A. (1995). El discurso académico como un discurso argumentativo. El argumento de autoridad en la primera clase de un curso académico. Comunicación, lenguaje, 25, 95-106.

- Cross-Alavedra, A. (2003). Convencer en clase. Argumentación y discurso docente. Barcelona: Ariel.

- Daly, A. y Unsworth, L. (2011). Analysis and comprehension of multimodal texts. En: Australian Journal of Language \& Literacy, 34 (1), 61-80.

- Del Caño, A. (1999). Los géneros orales informativos. En: Alcoba, Santiago y otros. (1999). La oralización. Barcelona, España: Editorial Ariel, S.A.

- Dolz, J. (1993). La interacción de las actividades orales y escritas y la enseñanza de la argumentación. Comunicación, lenguaje y educación, 23, 17-27

- Dolz, J. (1994). La interacción de las actividades orales y escritas en la enseñanza de la argumentación. Comunicación lenguaje y educación, 23, 17-27.
- Dolz, J. (1995). Escribir textos argumentativos para mejorar su comprensión. En: A. Camps y J. Dolz (1995).Comunicación, lenguaje y Educación, 26, 65-77.

- Dolz y Camps (1995). Introducción: Enseñar a argumentar un desafío para la escuela actual. Comunicación lenguaje y educación, 28, 5-8.

- Dolz, J. y Pasquier, A. (1996). Un decálogo para enseñar a escribir. Cultura y educación, 8(11), $31-41$.

- Dolz, J. (2011). Claves para enseñar a escribir. Leer.es.

- Dove, I. (2012). On Images as Evidence and Arguments. En: Van Emeren, F. y Garssen, B. (Eds). Topical Themes in Argumentation Therory. Twenty Exploratory Studies. Argumentation library, 22. (pp. 223-238) Dordrecht: Springer.

- Ducrot, O. y Anscombre, O. (1994). La argumentación en la lengua. España: Gredos.

- Felton, M. y Kuhn, D. (2001). The Development of Argumentive Discourse Skill. Discourse processes, 32(2-3), 135-153.

- Fisher, G. (2005). Federal rules of evidence: Statutory and case supplement. New York: Foundation Press.

- $\quad$ Fleming, D. (1996). Can pictures be arguments? Argumentation and advocacy, 33(1), 11-22.

- Golanics, J. y Nussbaum, E. (2007). Enhancing online collaborative argumentation through question elaboration and goal instructions. Journal of Computer Assisted Learning. 24, 167-180.

- Groarke, L. (1996). Logic, art and argument. Informal Logic, 18(2-3) ,105-131.

- Halliday, M. A (1978). El lenguaje como semiótica social. La interpretación del lenguaje y del significado, México: Fondo de la cultura económica.

- Jewitt, C. (2002). The move from page to screen: the multimodal reshaping of school English. Journal of Visual Communication, 1(2), 171-196.

- Jewit, C. (2008). Multimodal Discourses across the Curriculum. En: M. Martin-Jones, A. M. de Mejia and N. H. Hornberger (eds), Encyclopedia of Language and Education, 2nd Edition, Discourse and Education, 3, 357-367.

- Jiménez-Aleixandre, M.; Rodríguez, B., y Duschl, A. (Eds.) (2000). "Doing the Lesson" or "Doing Science": Arguments in High School Genetics. Science Education 84, 757-792. 
- Jiménez-Aleixandre, M. Py Díaz de Bustamante, J. (2003). Discurso de aula y argumentación en la clase de ciencias: cuestiones teóricas y metodológicas. Enseñanza de la Ciencias, 21 (3), $359-370$.

- Jiménez-Aleixandre, M. P y Erduran, S. (2007). Argumentation in Science Education Perspectives from Classroom-Based Research. Springer Science.

- Kress, G. (1977). Tense as modality. UEA Papers in Linguistics, 5, 40-50

- Kress, G. (1987). Communication and Culture: An Introduction. Sydney, University of New South Wales Press.

- $\quad$ Kress, G. y van Leeuwen, T. (1996). Reading images: The grammar of graphic design. London: Routledge.

- Kress, G. (2005). Alfabetismo y multimodalidad. Un marco teórico. En G. Kress, El alfabetismo en la era de los nuevos medios de comunicación (49-82). Granada, España: Ediciones Aljibe.

- Kress, G. y Benzemer, J. (2008). Writing in multimodal texts: A social semiotic account of designs for learning. Written Communication, 25(2), 166-195.

- Kuhn, D. (1991). The skills of argument. Cambridge: University Press.

- Leitão, S. (2000). The Potential of Argument in Knowledge Building. Human Development, 43, 332-360.

- Martí, E. (2012). Cognición y semiosis. En: Carretero, M y Castorina, J.A. (2012 comps). Desarrollo cognitivo y educación [II]. Procesos del conocimiento y contenidos específicos, Buenos Aires: Paidós.

- Martínez-Solís, M. (2002). Estrategias de lectura y escritura de textos. Colombia: Universidad Del Valle.

- Marinkovich, J. (2007). Las estrategias cognitivo-retóricas y la dimensión dialéctica de la argumentación oral en una clase de lengua castellana y comunicación. Revista Signos, 40 (63), 127-146.

- Ministerio de Educación Nacional -MEN(2003). Estándares curriculares para el área de lenguaje. Bogotá: Editorial Magisterio.

- Monsalve-Upegui, M. E. (2013), Habilidades argumentativas en la escritura de textos multimodales. Trabajo de Investigación para optar al título de Magíster en Educación. Medellín: Universidad de Antioquia. Dirigió cómo Tutor: Dora Inés Chaverra F y Wilson Bolívar.
- New London Group. (1996). A pedagogy of multiliteracies: Designing social futures. Harvard Educational Review, 66(1), 60-92.

- Nettel, A. L. (2005). The power of image and the image power: The case of law. Word and image, 21(2), 137-150.

- Noroozi, O.; Weinberger, A.; Biemans, J.A.; Mulder, M.; y Chizari, M. (2013) Facilitating argumentative knowledge construction through a transactive discussion script in CSCL. Computers \& Education, 61, 59-76.

- Nussbaum E. M. y Bendixen L. D. (2005). Approaching and avoiding arguments: The role of epistemological beliefs, need for cognition, and extraverted personality traits. Contemporary Educational Psychology, 28(4), 573-595.

- Parodi-Sweis, G. (2000). La evaluación de la producción de textos escritos argumentativos: una alternativa cognitivo/discursiva. Revista signos, 33(47), 151-166.

- Perelman, Ch. (1977). El imperio retórico: retórica y argumentación. Cap. II, la argumentación, el orador y su auditorio. 29-41. Barcelona: Grupo editorial norma.

- Perelman, Ch. y Olbrechts-Tyteca, L. (2001). Tratado de la argumentación. La nueva retórica. Madrid, España: Gredos. Traducción española de Julia Sevilla Muñoz.

- Perelman, F. (2001). Textos argumentativos: su producción en el aula. Lectura y Vida, 22 (2), 32-45.

- Poblete-Olmedo, C. (2005). Producción de textos argumentativos y metacognición. Letras, 47(71), 63-88.

- Plantin, C. (1998). La argumentación. España: Ariel.

- Ramírez-Guzmán, D.P. (2012). Argumentación y TIC en la escuela: Hacia la construcción colaborativa de argumentos. Trabajo de Investigación para optar al título de Magíster en Educación. Medellín: Universidad de Antioquia. Dirigió cómo Tutor: Wilson Bolívar.

- Roque, G. (2012). Visual Argumentation: A Further Reappraisal. En: Van Emeren, F... y Garssen, B. (Eds). Topical Themes in Argumentation Therory. Twenty Exploratory Studies. Argumentation library, 22. (273-288) Dordrecht: Springer.

- Rubio, M. y Arias, V. (2002). Una secuencia didáctica para la enseñanza de la argumentación escrita en el Tercer Ciclo. Lectura y vida, 23(4), 34-41. 
- Sánchez, I., y Álvarez, N. (2001). El discurso argumentativo de los escolares venezolanos. Cali: Cátedra UNESCO para la lectura y la escritura en América Latina.

- Stein, P. (2000). Rethinking Resources in the ESL Classroom: Rethinking Resources: Multimodal Pedagogies in the ESL Classroom. TESOL Quarterly, 34(2), 333-336.

- Stein, P. (2008a). Multimodal Instructional Practices. In J. Coiro, M. Knobel, C. Lankshear y D. Leu. Handbook of Research on New Literacies (871-898). New York: Lawrence Erlbaum Associates.

- Stein, P. (2008b). Multimodal Pedagogies in Diverse Classrooms: Representation, rights and resources, Chapter 6 Multimodal pedagogies: instances of practice. $121-152$.

- $\quad$ Shin-shin, D y Cimasko, T. (2008). Multimodal Composition in a College ESL Class: New Tools, Traditional Norms. Computers and Composition 25, 376-395.

- Tamayo-Alzate, O. E (2013). La argumentación como constituyente del pensamiento crítico en los niños. Hallazgos, 9(17), 211-233.

- Toulmin, S.(1958). Losusos delaargumentación. Barcelona: Ediciones península.

- Van den Hoven, P. (2012). The Narrator and the Interpreter in Visual and Verbal Argumentation. En: Van Emeren, F. y Garssen, B. (Eds). Topical Themes in Argumentation Therory. Twenty Exploratory Studies. Argumentation library, 22. (257-271) Dordrecht: Springer.

- Van Eemeren, F., Grootendorst, R. (2002). Argumentación, comunicación y falacias. Una perspectiva pragmadialéctica. Ediciones Universidad Católica de Chile: Chile.
- Veerman A. (2003). Constructive discussions through electronic dialogue. In Arguing to Learn: Confronting Cognitions En: ComputerSupported Collaborative Learning Environments (Eds J. Andriessen, M. Baker y D. Suthers), 117-143. Boston: Kluwer.

- Voss, J. F., Blais, J., Mary L., Means, M., Greene, T., y Ahwesh. E. (1986). Informal Reasoning and Subject Matter Knowledge in the Solving of Economics Problems by Naive and Novice Individuals. Cognition and Instruction, 3(4), 269302.

- Voss, J. F., Wiley, J., y Sandak, R. (1999). Reasoning in the Construction of Argumentative Texs. En: Foaundation of Argumentative text Processing. Eds J. Andriessen y C. Pierre). 29-40. Amsterdam: University Press.

- Ward, S. y Tiessen, E. (1997). Adding educational value to the Web: active learning with a live page. Educational Technology, 22-30.

- Weston, A. (2003). Las claves de la argumentación. Barcelona: Ariel S. A.

- Wikan, G., Mølster, T., Faugli, B. y Hope, R. (2010). Digital multimodal text and their role in project work: opportunities and dilemmas. Technology, Pedagogy and Education, 19(2), 225-235.

- Weinberger, A., Stegmann, K., y Fischer, F. (2007). Knowledge convergence in collaborative learning: Concepts and assessment. Learning and Instruction, 17(4), 416-426.

- Weinberger,A. y Fischer, F (2006).Aframework to analyze argumentative knowledge construction in computer-supported collaborative learning. Computers \& Education, 46(1), 71-95. 